\title{
Different subtypes of pseudohypoparathyroidism in the same family with an unusual psychiatric presentation of the index case
}

\author{
A J Pollard, M Prendergast, F Al-Hammouri, P H W Rayner, N J Shaw
}

\begin{abstract}
A 13 year old Asian girl presenting with apparent hysterical paralysis and subsequent rapid cycling bipolar mood disorder was found to have biochemical evidence of pseudohypoparathyroidism type II. The mood disorder responded to treatment of the pseudohypoparathyroidism with a vitamin $D$ analogue. Investigation of her parents and siblings showed phenotypes consistent with two distinct types of pseudohypoparathyroidism (type I and type II) in different family members.

(Arch Dis Child 1994; 70: 99-102)
\end{abstract}

Pseudohypoparathyroidism comprises a heterogeneous group of genetically determined disorders which are still to be fully characterised in molecular and genetic terms. Pseudohypoparathyroidism presents with biochemical evidence of parathyroid hormone resistance, which is shown clinically by deficient phosphaturic and calcaemic responses to exogenous parathyroid hormone.

We report an unusual psychiatric presentation of a child with pseudohypoparathyroidism and describe the clinical and biochemical features of other members of the child's family.

\section{Case report}

An Asian girl aged 13.3 years, G, presented to Birmingham Children's Hospital unable to walk, with total alopecia, poor appetite, and weight loss of $6 \mathrm{~kg}$. She was well dressed, calm and relaxed, and indifferent to her hair loss and the loss of function in her legs. She had recently been an inpatient at another hospital with a diagnosis of hysterical paralysis. She was admitted the following week for investigation, at which time she was walking and physical examination was normal, though weight was below the third centile (height 25th-50th centile) and her head had clearly been shaved. She spoke English fluently and intelligently and she seemed indifferent ('la belle indifference') to the events leading up to her admission. Her mental state was otherwise normal.

Her past history was unremarkable. Her father was a 40 year old packer who was unable to work because of vertigo and agoraphobia. He regularly attended a psychiatric outpatient clinic with depression and had had febrile convulsions as an infant. The child's mother (A) had previously had tuberculosis and was also unemployed. The marriage was consan- guineous. The child was engaged to a man in Pakistan.

The child was the fourth of eight children aged 3-21 years. The three youngest and the eldest had all had recurrent (probably febrile) convulsions, the eldest having had more than 10 fits as a child. The third youngest, E, a boy aged 10 , had testicular feminisation syndrome and was initially raised as a girl (to the age of 5 years). $\mathrm{He}$ is thought by the family to have magical powers.

The child's headteacher telephoned personally with a school report. He said that, until a month before admission, $G$ had been a model pupil but recently she had become unpopular and had started to tell bizarre stories of her home life.

Later during her admission, prominent mood swings from hour to hour and day to day were noted. At times she dressed in Western clothes and was found dancing alone enthusiastically; later the same day she changed into dull cultural clothes and wandered with her head bowed and covered looking miserable. Manchester depression and mania nurse rating scales ${ }^{1}$ were performed, which supported the clinical impression of significant mood swings even within the same day, and a diagnosis of rapid cycling bipolar (manic-depressive) mood disorder was made.

\section{Results}

Investigations, including electrolytes, full blood cell count, creatinine, erythrocyte sedimentation rate, thyroid function tests, lead, zinc, magnesium, copper, renal ultrasound, computed tomography of the head and electroencephalography were normal. There was, however, an abnormality of calcium metabolism (normal range): serum calcium 1.82 $\mathrm{mmol} / \mathrm{l}(2 \cdot 15-2.70 \mathrm{mmol} / \mathrm{l})$, phosphate 1.79 $\mathrm{mmol} / \mathrm{l}(1.0-1.8 \mathrm{mmol} / \mathrm{l})$, alkaline phosphatase $726 \mathrm{U} / 1(275-800 \mathrm{U} / \mathrm{l})$, parathyroid hormone $269 \mathrm{ng} / 1$ (10-40 ng/1), and 25-hydroxyvitamin D $2.4 \mathrm{ng} / \mathrm{ml}(3-30 \mathrm{ng} / \mathrm{ml})$. The high phosphate level and normal alkaline phosphatase, despite greatly increased parathyroid hormone and hypocalcaemia, suggested pseudohypoparathyroidism and a parathyroid hormone infusion test $^{2}$ was arranged.

After treatment with a vitamin $\mathrm{D}$ analogue and establishment of normocalcaemia, parathyroid hormone infusion (200 IU intravenously) gave a normal increase in urinary excretion of cyclic adenosine monophosphate (cAMP) but a blunted phosphaturic response (see figs 1 and 2) consistent with type II pseudohypoparathyroidism. 


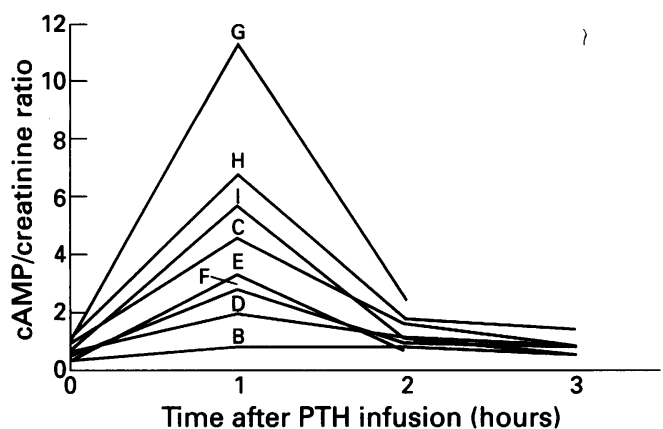

Figure 1 Renal $c A M P$ uric response to intravenous infusion of parathyroid hormone (PTH) in eight member of the same family. One subject (B) shows no $c A M P$ Puric response and has type $I b$ pseudohypoparathyroidism.

These findings prompted investigation of the other immediate family members with calcium, parathyroid hormone, and parathyroid hormone infusion tests (see figs 1 and 2). G's father (B) had normal calcium metabolism but showed no increase in cAMPuria or phosphaturia with parathyroid hormone infusion, suggesting type I pseudohypoparathyroidism. G's sisters F (12 years) and $H$ (14 years) had the same blunting of phosphate excretion but a normal increase in cAMP with parathyroid hormone infusion, consistent with a diagnosis of type II pseudohypoparathyroidism. $\mathrm{H}$ was normocalcaemic; $\mathrm{F}$ was hypocalcaemic $(1.8 \mathrm{mmol} / \mathrm{l})$ initially, but her serum calcium later returned to normal without treatment. These girls were also above the 97th centile for weight, had greatly increased parathyroid hormone concentrations, and were not deficient in vitamin $\mathrm{D}$.

There were good biochemical and clinical responses to treatment with a vitamin D analogue. The mood swings disappeared almost at once and $\mathrm{G}$ returned to school and was re-elected form captain by her peers.

\section{Discussion}

In the original description, Albright et al described three patients with hypocalcaemia,

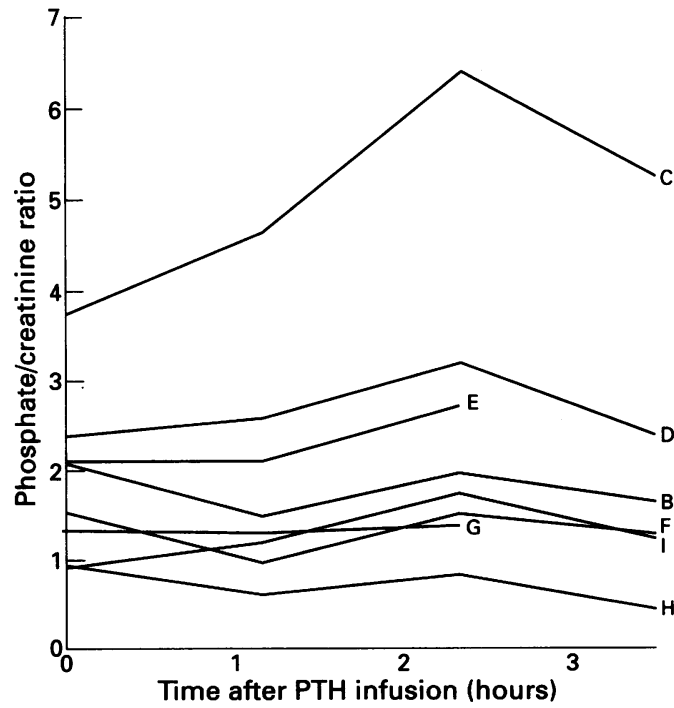

Figure 2 Renal phosphaturic response to intravenous infusion of parathyroid hormone (PTH) in eight member of the same family. Four subjects showed no phosphaturic response $(B, G, F, H$; see text) and have

pseudohypoparathyroidism. hyperphosphataemia, and normal renal function who had characteristic clinical features of obesity, short stature, round face, and short metacarpals, and reduced responses to exogenous parathyroid hormone. ${ }^{3}$ It is now apparent that some subjects with this phenotype (Albright's hereditary osteodystrophy) have normal calcium metabolism, considered by Albright et al as a distinct syndrome, pseudopseudohypoparathyroidism. ${ }^{4}$

Pseudohypoparathyroidism is divided into two groups depending on the presumed site of resistance to parathyroid hormone. In the normal subject, infusion of exogenous parathyroid hormone results in increased urinary excretion of phosphate and cAMP. In type I pseudohypoparathyroidism there is no cAMPuria in response to parathyroid hormone infusion and in type II cAMP excretion is increased in the basal state and there is a normal response to parathyroid hormone infusion. In types I and II pseudohypoparathyroidism the renal phosphaturic response to parathyroid hormone infusion is blunted.

Type I pseudohypoparathyroidism can be further subdivided. In type Ia pseudohypoparathyroidism the defect is in the Gs membrane protein which couples the parathyroid hormone receptor to the cAMP 'intracellular messenger'. These patients have the Albright's hereditary osteodystrophy phenotype and may be hypocalcaemic or normocalcaemic (pseudopseudohypoparathyroidism). Resistance to other hormones which use the Gs protein-cAMP messenger system may coexist in these patients.

The molecular defect in type Ib pseudohypoparathyroidism is heterogenous, involving biologically inactive parathyroid hormone or abnormalities in the parathyroid hormone receptor itself. These patients may have normal phenotypes or Albright's hereditary osteodystrophy phenotypes.

Patients with type II pseudohypoparathyroidism have a normal increase in cAMPuria in response to parathyroid hormone infusion, deficient phosphaturic responses, and a normal phenotype (see fig 3 ).

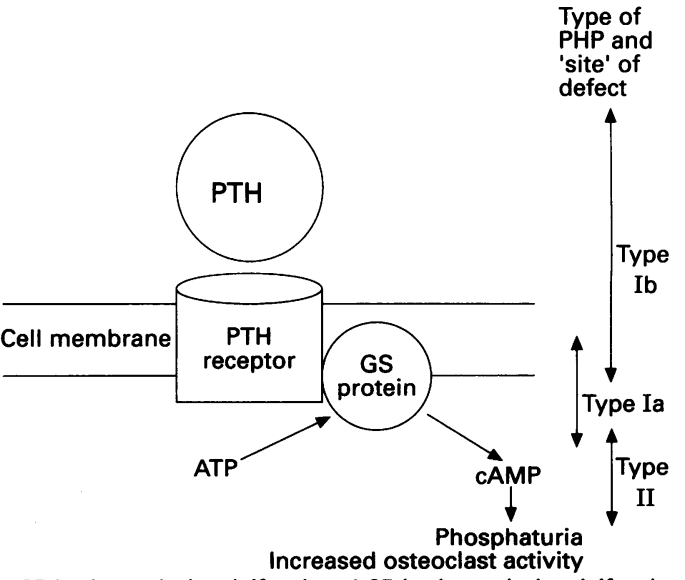

25-hydroxycholecalciferol $\rightarrow$ 1,25-hydroxycholecalciferol

Figure 3 Interaction between parathyroid hormone (PTH) and its receptor producing the intracellular $(P T H)$ and its receptor producing the intracelluh
second messenger $C A M P$ and sites of defect in pseudohypoparathyroidism $(P H P)$. GS protein = protein coupling $P T H$ receptor to $c A M P ; c A M P=$ intracellular 'messenger'. 


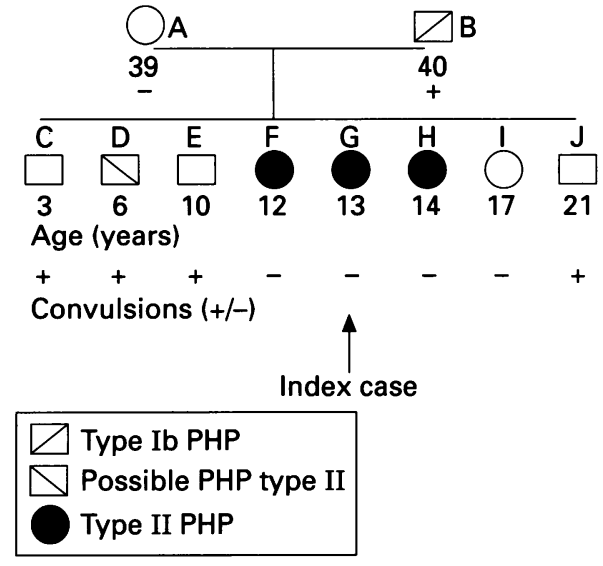

Figure 4 Family tree showing subjects affected by pseudohypoparathyroidism (PHP).

Our patient, G, had hypocalcaemia, normophosphataemia, normal alkaline phosphatase activity, and increased parathyroid hormone. The normal cAMPuria but blunted phosphaturia with parathyroid hormone infusion is consistent with type II pseudohypoparathyroidism.

Findings compatible with type II pseudohypoparathyroidism have been described in patients with vitamin $\mathrm{D}$ deficiency ${ }^{6}$ but treatment with vitamin $\mathrm{D}$ in these patients restored a normal phosphaturic response to pseudohypoparathyroidism. Our patient was shown to be vitamin $\mathrm{D}$ deficient at presentation, but underwent parathyroid hormone infusion after more than one month of treatment with a vitamin $\mathrm{D}$ anologue, by which time she was normocalcaemic.

There is only one previously published description of familial pseudohypoparathyroidism type II (in two brothers). ${ }^{6}$ In our sibship there were three sisters with characteristic pseudohypoparathyroidism type II responses to infused parathyroid hormone $(F$, $\mathrm{G}, \mathrm{H}$ ) (fig 4).

The father in this family group (B) had total loss of olfaction and was noted to be overweight ( $>97$ th centile), both features of pseudohypoparathyroidism, and showed responses to infused parathyroid hormone consistent with type I pseudohypoparathyroidism (blunted cAMPuria and phosphaturia), but did not have the Albright's hereditary osteodystrophy phenotype, suggesting type Ib pseudohypoparathyroidism. Our current understanding of pseudohypoparathyroidism suggests that type Ib and type II pseudohypoparathyroidism are genetically distinct disorders, and if this is so it is difficult to explain the diversity in this family. The probable candidate gene in type Ia pseudohypoparathyroidism has been assigned ${ }^{8}$ to chromosome 20q12-q13.2 and the defect in the Gs protein is well documented. The molecular and genetic defects in type $\mathrm{Ib}$ and type II pseudohypoparathyroidism are certainly heterogeneous and not yet clarified. In the future further studies using cDNA probes when available would be helpful in this family group.

Our index case presented with overt clinical and biochemical evidence of disease but had previously been well. In addition, other members of the family with biochemical evidence of pseudohypoparathyroidism had no clinical signs nor symptoms. Factors precipitating overt symptoms in subjects with subclinical pseudohypoparathyroidism are unknown, but vitamin $\mathrm{D}$ deficiency might be a candidate in our family. It is unclear, though possible, that the father's psychiatric history was related to his abnormal calcium metabolism (type Ib pseudohypoparathyroidism). Overt pseudohypoparathyroidism has been described in a 34 year old woman with previous subclinical type II pseudohypoparathyroidism, presenting during the second half of pregnancy. ${ }^{9}$

Resistance to hormones other than parathyroid hormone, including thyroid, gonadotrophins, prolactin, and glucagon is well described in type Ia (Albright's hereditary osteodystrophy phenotype) pseudohypoparathyroidism and screening for hypothyroidism is mandatory in these children, but it is not classically described in type Ib or type II pseudohypoparathyroidism as seen here.

It is interesting to note that the father and four of the siblings had recurrent convulsions in early childhood. Pseudohypoparathyroidism presenting with convulsions has been described ${ }^{10}$ and neuromuscular symptoms secondary to hypocalcaemia such as paresthesias, tetany, and seizures (and prolonged QT interval on electrocardiogram) are well described in pseudohypoparathyroidism. ${ }^{5}$

The association between hypoparathyroidism (surgical and idiopathic) and psychiatric symptoms, such as delirium, poor concentration, emotional lability and impaired intellectual function, is well recognised. ${ }^{11}$ Parasthesias, tetany, and seizures are recognised features of pseudohypoparathyroidism ${ }^{5}$ and the former may have contributed to the apparent hysterical paralysis in our index case.

Some published work suggests a particular relation between bipolar affective disorders and intracellular calcium metabolism, though there is no published study of children. Dubovsky et al ${ }^{12}$ showed increased mean intracellular calcium ion concentrations in lymphocytes and platelets of bipolar patients compared with controls and further showed normal platelet intracellular calcium ion concentrations in euthymic treated bipolar patients. ${ }^{13}$ In a review of calcium status in 29 unipolar and 14 bipolar depressed patients Bowden et al found lower plasma calcium concentrations than in normal controls. ${ }^{14}$ These findings have led to the therapeutic use of calcium antagonists in bipolar affective disorders with claims of therapeutic efficacy. ${ }^{15} 16$ It has also been suggested that the action of lithium in the treatment of mood disorders may be mediated by its blocking of inositol1,4,5-triphosphate, an intracellular second messenger, which affects the increase in ionic intracellular calcium in secretory cells triggering neurotransmitter release. ${ }^{17}$

Although the intracellular calcium concentration in pseudohypoparathyroidism is low, ${ }^{18}$ clouding the relation between the findings in 
other studies of bipolar mood disorder and our patient, it nevertheless seems likely that the psychiatric symptoms and their rapid resolution in our patient may be attributable to the disorder in calcium metabolism and its subsequent treatment.

Although mental retardation is common in type Ia (Albright's hereditary osteodystrophy phenotype) pseudohypoparathyroidism, psychiatric disturbance is not well described. There is a published example of periodic psychosis in pseudopseudohypoparathyroidism in a subject with the classic phenotype. ${ }^{19}$

This case report draws attention to the possibility of underlying disorders of calcium metabolism in psychiatric presentations in childhood. It is the second description of familial type II pseudohypoparathyroidism. The coexistence of type Ib pseudohypoparathyroidism in the same family challenges the current classification of this group of disorders.

We acknowledge the assistance of the nursing staff on Ward 7 at Birmingham Children's Hospital for completing the department of clinical chemistry for biochemical analysis.

1 Brierley CE, Szabadi E, Rix KJB, Bradshaw CM. The Manchester nurse rating scales for the daily simultaneous assessment of depressive and manic ward behaviours. $\mathscr{F}$ Affective Disord 1988; 15: 45-54.

2 Hughes IA. Calcium, parathyroid and vitamin D. Handbook of endocrine tests in children. Vol 6. Bristol: Wright, 1986: $74-7$.

3 Albright $\mathrm{F}$, Burnett $\mathrm{CH}$, Smith $\mathrm{PH}$, Parson W. Pseudohypoparathyroidism - an example of SeabrightBantam syndrome Endocrinology 1942; 30: 922.

4 Albright F, Forbes AP, Henneman PH. Pseudopseudohypoparathyroidism. Trans Assoc Am Physician 1962; 65: 337 .

5 Spiegel AM. Pseudohypoparathyroidism. In: Scriver CR Beaudet AL, Sly WS, Valle D. Metabolic basis of inherited disease. 6th Ed. Vol 79. New York: McGraw Hill, 1989: 2013-27

6 Rao DS, Parfitt AM, Kleerekoper M, Pumo BS, Frame B. Dissociation between the effects of endogenous parathyroid hormone on adenosine 3,5-monophosphate generation and phosphate reabsorption in hypocalcaemia due to vitamin D depletion: an acquired disorder resembling vitamin D depletion: an acquired disorder resembling pseudohypoparathyro

7 Matsuda I, Takekoshi Y, Tanaka M, Matsuura N, Nagai B, Seino Y. Pseudohypoparathyroidism type II and anticonvulsant rickets. Eur $\mathcal{F}$ Pediatr 1979; 132: 303.

8 Rao VV, Schnittger S, Hansmann I. G protein Gs alpha (GNAS 1), the probable candidate gene for Albright hereditary osteodystrophy, is assigned to human chromosome 20q12-q13.2 Genomics 1991; 10: 257-61.

9 Saito H, Saito M, Saito K, et al. Subclinical pseudohypoparathyroidism type II: evidence for failure of physiopoparathyroidism type II: evidence for failure of physiological adjustment in calcium metabolisn

10 Bonadio WA. Hypocalcaemia caused by pseudohypoparathyroidism presenting as convulsion. Pediatr Emerg Care 1989; 5: 22-3.

11 Denko JD, Kaelbling R. The psychiatric aspects of hypoparathyroidism. Acta Psychiatr Scand 1962; suppl 164: $1-70$.

12 Dubovsky SL, Murphy J, Thomas M, Rademacher J. Abnormal intracellular calcium ion concentration in platelets and lymphocytes in bipolar patients. $A m \mathcal{F}$ Psychol 1992; 149: 118-20.

13 Dubovsky SL, Lee C, Christiano J, Murphy J. Elevated platelet intracellular calcium concentration in bipolar platelet intracellular calcium concentration
depression. Biol Psychol 1991; 29: 441-50.

14 Bowden CL, Huang LG, Javors MA, et al. Calcium function in affective disorders and healthy controls. Biol Psychol 1988; 23: 367-76.

15 Mathis P, Scmitt L, Moron P. Efficacy of verapamil in mania crises. Encephale 1988; 14: 127-32.

16 Manna V. Bipolar affective disorders and role of intraneuronal calcium. Therapeutic effects of the treatment with lithium salts and/or calcium antagonists in patients with rapid polar inversion. Minerva Med 1991; 82: 757-63.

757-63. CI CL. Effects of lithium on platelet ionic intracellular calcium concentration in patients with bipolar (manicdepressive) disorder and healthy controls. Life Sci 1990;

18 Hosking DJ, Kerr D. Mechanisms of parathyroid hormone resistance in pseudohypoparathyroidism. Clin Sci 1988; 74: $561-6$.

19 Furukawa T. Periodic psychosis associated with pseudopseudohypoparathyroidism. F Nerv Ment Dis 1991; 179: 637-8. 